\title{
The Association of Combined Total Kidney and Liver Volume with Pain and Gastrointestinal Symptoms in Patients with Later Stage Autosomal Dominant Polycystic Kidney Disease
}

\author{
Hedwig M.A. D’Agnolo ${ }^{a}$ Niek F. Casteleijn ${ }^{c}$ Tom J.G. Gevers ${ }^{a}$ Hans de Fijter ${ }^{\mathrm{e}}$ \\ Maartje D.A. van Gastel ${ }^{d}$ Annemarie L. Messchendorp ${ }^{d}$ Dorien J.M. Peters ${ }^{f}$ \\ Mahdi Salih $^{g}$ Darius Soonawala $^{\mathrm{e}}$ Edwin M. Spithoven ${ }^{d}$ Folkert W. Visser $^{d}$ \\ Jack F.M. Wetzels ${ }^{b}$ Robert Zietse ${ }^{g}$ Ron T. Gansevoort ${ }^{d}$ Joost P.H. Drenth ${ }^{a}$ \\ a Department of Gastroenterology and Hepatology, and ${ }^{\mathrm{b}}$ Department of Nephrology, Radboud University Medical \\ Center Nijmegen, Nijmegen, ' Department of Urology, and ${ }^{\mathrm{d}}$ Department of Nephrology, University Medical Center \\ Groningen, University of Groningen, Groningen, ${ }^{\mathrm{e}}$ Department of Nephrology, and ${ }^{\mathrm{f}}$ Department of Human Genetics, \\ Leiden University Medical Center, Leiden, and ${ }^{9}$ Department of Nephrology, Erasmus Medical Center Rotterdam, \\ Rotterdam, The Netherlands
}

\section{Keywords}

Autosomal dominant polycystic kidney disease · Polycystic liver disease · Pain · Gastrointestinal symptoms · Total kidney liver volume

\begin{abstract}
Background: There is an ongoing debate if and how kidney and liver volume are associated with pain and gastrointestinal (Gl) symptoms in autosomal dominant polycystic kidney disease (ADPKD) patients. Since both kidney and liver volume could interact, we investigated whether combined total kidney and liver volume had stronger associations with ADPKDrelated pain and $\mathrm{Gl}$ symptoms than the volumes of the organs separately. Methods: We used baseline data from the DIPAK-1 study, which included ADPKD patients with an estimated glomerular filtration rate (eGFR) between 30 and $60 \mathrm{~mL} /$ $\mathrm{min} / 1.73 \mathrm{~m}^{2}$. MR imaging was performed to measure heightadjusted total kidney volume (hTKV), height-adjusted total liver volume (hTLV) and the combination of both (height-adjusted total kidney liver volume [hTKLV]). Results: Three hun-
\end{abstract}

(c) 2017 S. Karger AG, Basel dred nine ADPKD patients were included with a mean age of $48 \pm 7$ years, $53 \%$ female, eGFR $50 \pm 11 \mathrm{~mL} / \mathrm{min} / 1.73 \mathrm{~m}^{2}$ and median hTKV, hTLV and hTKLV of 1,095 (758-1,669), 1,173 $(994-1,523)$ and $2,496(1,972-3,352) \mathrm{mL} / \mathrm{m}$, respectively. ADPKD-related pain and Gl symptoms were present in, respectively, 27.5 and $61.2 \%$ of patients. Gender was no effect modifier in the association between kidney and/or liver volume, and symptom burden, indicating that all models could be tested in the overall study population. hTKLV and hTLV were significantly associated with pain and Gl symptoms, whereas hTKV was not. Model testing revealed that the associations of pain and Gl symptoms with hTKLV were significantly stronger than with hTKV $(p=0.04$ and $p=0.04$, respectively) but not when compared to hTLV ( $p=0.2$ and $p=0.5$, respectively). Conclusions: This study indicates that combined kidney and liver volume was associated with the presence and severity of pain and GI symptoms in ADPKD, with a more prominent role for $\mathrm{hTLV}$ than for $\mathrm{hTKV}$.

(C) 2017 S. Karger AG, Basel

H.M.A.D. and N.F.C. contributed equally.

\section{KARGER}

E-Mail karger@karger.com

www.karger.com/ajn
Joost P.H. Drenth, MD, PhD

Professor of Gastroenterology and Hepatology

Department of Gastroenterology and Hepatology, Radboud University Medical Center PO Box 9101, NL-6500 HB Nijmegen (The Netherlands)

E-Mail joostphdrenth@ cs.com 


\section{Introduction}

Autosomal dominant polycystic kidney disease (ADPKD) is characterized by progressive renal cyst formation and the majority of patients also have liver cysts (>94\%) [1]. During one's lifetime, kidney and liver volume increase, leading to the distension of the renal and hepatic capsules, and compression of adjacent organs [2]. Consequently, a substantial proportion of ADPKD patients experiences pain and gastrointestinal (GI) symptoms, such as abdominal fullness and early satiety [3-6].

There is an ongoing debate if and how kidney and liver volume are associated with pain and GI symptoms. A number of studies have investigated symptom burden in ADPKD patients [5, 7-9]. The largest of these studies did not find an association between kidney volume and pain, except for a small subgroup with very large kidneys [5]. Another study concluded that quality of life was not different between patients with a total kidney volume (TKV) larger or smaller than $1,000 \mathrm{~mL}$, but the effect of liver volume was not assessed [8]. Two studies that analyzed the effect of liver volume on quality of life, showed conflicting results, with one study finding no relation and the other a significant, but weak association between liver volume and symptom burden $[10,11]$. Of note, all aforementioned studies varied in the use of height- or non-heightadjusted kidney and liver volumes [5, 7-10]. In terms of disease progression, height-adjusted TKV (hTKV) has been shown to be more closely related to the rate of disease progression than non-height-adjusted TKV [12]. The question arises whether the conflicting data in literature may be explained by the fact that sometimes heightand sometimes non-height-adjusted volumes were used to test correlations with symptom burden.

Another factor that potentially affects symptom burden is a difference in gender. In literature, females are overrepresented among cohorts of patients with symptomatic ADPKD $[13,14]$. This is usually attributed to the presence of a more severe liver phenotype in females [15]. On the other hand, pain sensitivity has been suggested to be greater among females, and females are more likely to report GI symptoms when compared to males [16-18]. To our knowledge, it has not been investigated whether higher symptom burden in females with ADPKD is caused by differences in reporting by gender in general, or by differences in kidney and/or liver size between both genders.

Since both kidney and liver volume drive intra-abdominal volume, it is reasonable to assess the association of combined kidney and liver volume with ADPKD-related pain and GI symptoms [19]. Therefore, we investi- gated in a large cohort of ADPKD patients whether combined kidney and liver volume is more strongly associated with ADPKD-related pain and GI symptoms than kidney or liver volume alone; second we investigated to understand whether there is a difference in the strength of this association between males and females, and third whether height-adjusted volumes are more strongly associated with pain and GI symptoms than non-heightadjusted volumes.

\section{Materials and Methods}

\section{Patients and Study Design}

Baseline data was used from the DIPAK-1 study, an investigator-driven, multicenter, randomized, controlled clinical trial that included ADPKD patients with an estimated glomerular filtration rate (eGFR) between 30 and $60 \mathrm{~mL} / \mathrm{min} / 1.73 \mathrm{~m}^{2}$ and age 18-60 years. Patients were enrolled at 4 University Medical Centers in the Netherlands (Groningen, Leiden, Nijmegen and Rotterdam) between June 2012 and March 2015. ADPKD diagnosis was based on the modified Ravine criteria [20]. Exclusion criteria were among others, concomitant illnesses likely to confound the natural decline of renal function in ADPKD, for example, diabetes mellitus. Details of the study protocol have been published elsewhere [21]. The Medical Ethics Committee of the University Medical Center Groningen approved the protocol of the DIPAK-1 study that was conducted in accordance with the International Conference of Harmonization Good Clinical Practice Guidelines and in adherence to the ethics principles that have their origin in the Declaration of Helsinki (METc2012/060). All patients gave their written informed consent.

\section{Data Collection, Measurements and Definitions}

Evaluations were performed in all patients at baseline including standardized interviews, physical examination, collection of blood samples and MR imaging. During the interviews information was gathered about demographics, medical history, pain and GI symptoms. Renal pain was defined as pain or discomfort located in the flank, the lower back or abdomen that according to patients was caused by renal cysts. Liver pain was defined as pain or discomfort located in the right upper abdomen, behind or below the rib cage that according to patients' well-being is most likely to be caused by liver cysts. The severity of renal and/or liver pain during the last 4 weeks, including pain at baseline, was assessed on a $1-10$ scale $(1=$ no pain, $10=$ worst possible pain), and the presence of renal or liver pain was defined as a score $\geq 3$. Since it is difficult to distinguish between renal and liver pain, we used a composite score for ADPKD-related pain. We choose $\geq 3(3-10)$ as a cut-off point so that every patient with moderate to severe pain was included [22]. For severity of ADPKD-related pain, the highest score on either renal or liver pain was used. We also asked patients about the presence of renal pain in history, defined as renal or liver pain that occurred in the weeks, years or months prior to the 4 weeks prior to baseline.

The presence of GI symptoms over the last 4 weeks was recorded through the GI symptoms questionnaire [23] (online suppl. Table 1; for all online suppl. material, see www.karger.com/ doi/10.1159/000479436). This questionnaire contains 11 items 
such as upper and lower abdominal pain, early satiety and dyspnea. All symptoms were assessed using a 7-point Likert scale, ranging from 1 ("none") to 7 ("severe"). Symptom severity sum score was calculated by summing all scores and converting it to a score from 0 to 100 [23]. The reason to create a sum score for several GI symptoms is that ADPKD patients may encounter various complaints. Studying each complaint separately would result in a difficult message and would not correctly reflect the actual clinical picture of these patients, which is complex and consists of several complaints, that cumulatively determines disease burden. We chose to include both lower and upper abdominal pain in our analysis because we know from clinical experience that some patients have upper abdominal pain, whereas others have lower abdominal pain and some have both. The presence of GI symptoms was defined as a score of $\geq 3$ on at least one of $11 \mathrm{GI}$ symptoms. A score $\geq 3$ means that a symptom was at least moderately present. Serum creatinine was reported and used to eGFR (applying the Chronic Kidney Disease Epidemiology Collaboration equation) [24]. All patients underwent an MRI to assess kidney and liver volumes by the manually tracing method using the commercially available software Analyze Direct 11.0 (Analyze Direct, Inc., Overland Park, KS, USA). Kidney and liver volumes were calculated from the set of contiguous images by summing the products of the area measurements within the kidney or liver boundaries and slice thickness. Details of the imaging protocol have been reported previously [21]. hTKV, height-adjusted total liver volume (hTLV) and combined heightadjusted total kidney liver volume (hTKLV) were calculated as total organ volume in $\mathrm{mL}$ divided by height in meters.

\section{Statistical Analyses}

We performed a cross-sectional analysis of the baseline data of the DIPAK-1 study. Baseline characteristics were calculated for the overall population and stratified for patients experiencing ADPKD-related pain, experiencing GI symptoms and gender to objectify which patient characteristics were associated with pain or gastro-intestinal symptoms. Normally distributed variables are expressed as mean $\pm \mathrm{SD}$, whereas non-normally distributed variables are given as median and interquartile range (IQR). Differences in baseline characteristics between groups were calculated with a chisquare test for categorical data, and for continuous data with Student $t$ test or a Mann-Whitney $\mathrm{U}$ test in case of non-normally distributed data.

To investigate whether organ volume correlated (independent variable) with ADPKD-related pain and GI symptoms (dependent variables), univariate and multivariate linear regression analyses were performed. hTKV, hTLV, and hTKLV were logarithmic transformed to fulfill the requirement of normal distribution of the residuals for regression analysis. The multivariate linear analyses were subsequently adjusted for age and eGFR to correct for disease severity. To investigate differences between males and females the variable "gender" was added to the regression analysis. To explore whether associations between organ volume (i.e., hTKV, hTLV, and hTKLV) and symptom burden (i.e., ADPKD-related pain and GI symptoms) were different between males and females, interaction was tested by adding product terms (gender times volume) as independent variable to the analyses. In these univariate and multivariate linear regression analyses the complete study population, including all 309 patients with pain scores ranging from 1 to 10 , were included.

Bootstrapping is useful to investigate whether model " $\mathrm{X}$ " is more strongly correlated with independent variables compared to model "Y" that contains other independent variables. Therefore, we used bootstrapping (2,000 times) to investigate whether the association of hTKLV with ADPKD-related pain and GI symptoms was stronger than the associations of either hTKV or hTLV with ADPKD-related pain and GI symptoms [25]. In all regression analyses, we corrected for disease severity by adjustment for gender, age and eGFR. A sensitivity analysis was performed to test whether these associations were different in patients with larger kidneys (hTKV $>1,000 \mathrm{~mL} / \mathrm{m}$ ). This hTKV cut-off was based on findings in a previous observational study in ADPKD patients [5]. Lastly, bootstrapping was performed to analyze whether heightadjusted volume regression analysis were more strongly associated with pain and GI symptoms than non-height-adjusted regression analysis. All analyses were performed using SPSS software version 22.0 (Chicago, IL, USA) and STATA version 14 (StataCorp SE) statistical software, and a 2 -sided $p<0.05$ was considered to indicate statistical significance.

\section{Results}

\section{Patient Characteristics}

We enrolled 309 ADPKD patients, of which 53\% were female with a mean age of $48 \pm 7$ years. Following our inclusion criteria all patients had an impaired renal function with a mean eGFR of $50 \pm 11 \mathrm{~mL} / \mathrm{min} / 1.73 \mathrm{~m}^{2} . \mathrm{Me}-$ dian hTKV, hTLV and combined total kidney liver volume (hTKLV) were, respectively, 1,095 (758-1,669), $1,173(994-1,523)$, and 2,496 (1,972-3,352) $\mathrm{mL} / \mathrm{m}$. Liver cysts were present in a large majority of patients (93.2\%).

\section{ADPKD-Related Pain and GI Symptoms}

ADPKD-related pain was reported by $27.5 \%$ of the study population (renal pain: $24.9 \%$ and liver pain: $11.3 \%$; Table 1). Pain was more common in females than in males. Age and eGFR did not differ between patients with and without pain, while a history of renal pain, liver pain, urinary tract infection, renal cyst infection, liver cyst infection and macroscopic hematuria were more common in those who reported pain. Liver cysts were also more common in patients experiencing ADPKD-related pain. Larger hTLV and hTKLV were associated with pain, whereas hTKV was not. The median age at which patients experienced renal pain for the first time was 42 (IQR 3547 ) years. For liver pain this was 41 (IQR 34-49) years.

A total of $61.2 \%$ of the ADPKD patients experienced GI symptoms, with females being overrepresented in patients reporting these symptoms (Table 1). Age and eGFR were not different between patients with or without GI symptoms. The presence of GI symptoms was associated with a history of renal pain, liver pain, urinary tract infection, renal cyst infection and renal surgery. The most frequently 
Table 1. Baseline characteristics of DIPAK study participants stratified according to presence or absence of ADPKD-related pain and gastrointestinal symptoms

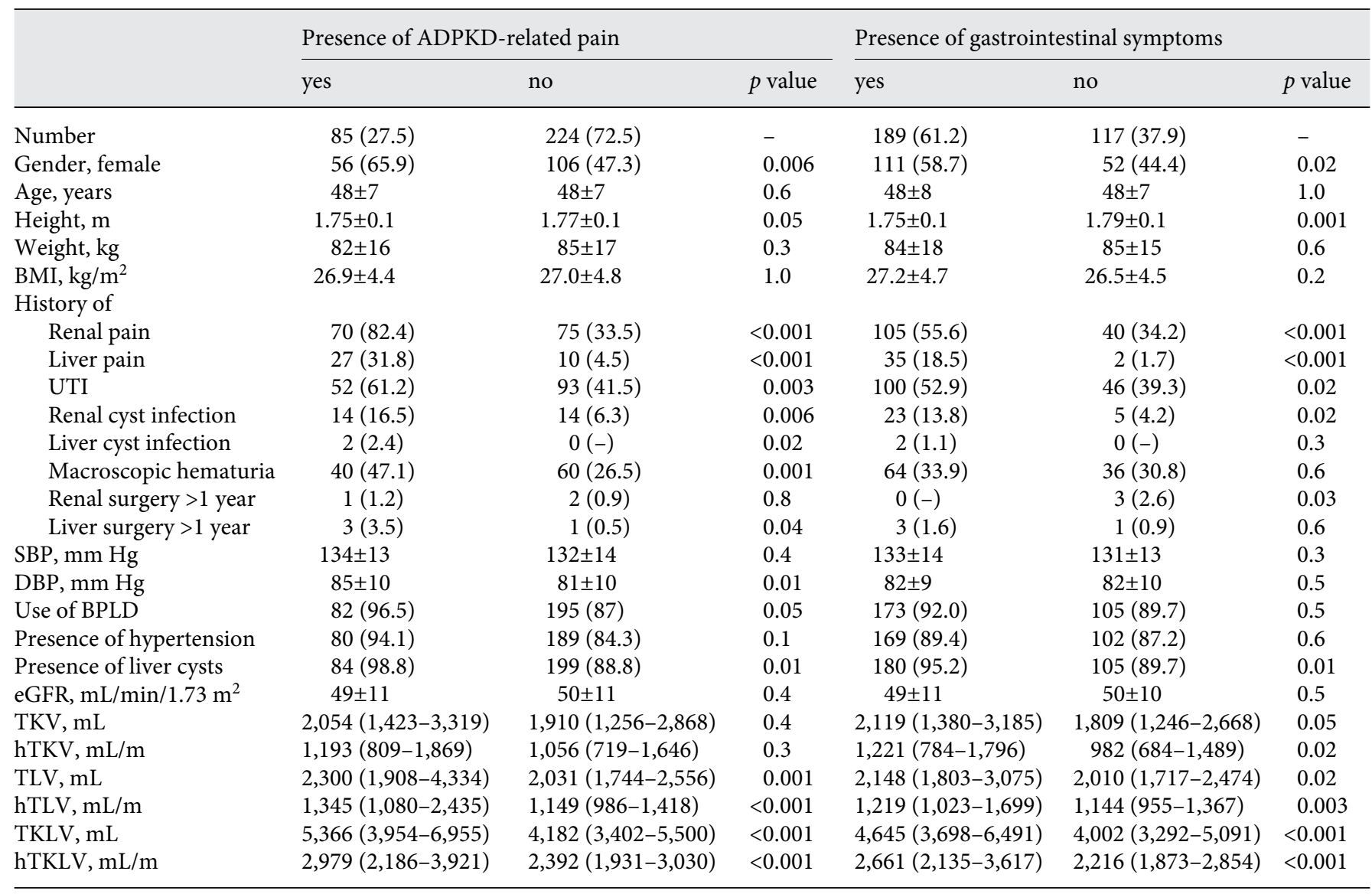

BMI, body mass index; UTI, urinary tract infection; SBP, systolic blood pressure; DBP, diastolic blood pressure; BPLD, blood pressure lowering drug; eGFR, estimated glomerular filtration rate; TKV, total kidney volume; hTKV, height-adjusted total kidney volume; TLV, total liver volume; hTLV, height-adjusted total liver volume; TKLV, total kidney liver volume; hTKLV, height-adjusted total kidney liver volume.

Data is shown as $n(\%)$, mean \pm SD or median (interquartile range). The denominator of each number in the table is the total number of patients in the column.

reported GI symptom was early satiety $(32.0 \%)$, followed by increased abdominal volume (25.2\%), dyspnea (24.6\%), heartburn (22.7\%) and regurgitation (18.4\%; Table 2).

Of note, 44 of the 309 patients (14.2\%) used mucosal protective and gastric antisecretory drugs. We observed that the use of these drugs was not associated with the GI sum score $(p=0.2)$. In addition, no difference was found in GI sum score between patients with and without using mucosal protective and gastric antisecretory drugs $(p=0.6)$.

\section{Association of Kidney and Liver Volume with Pain and GI Symptoms}

To investigate whether associations between volumes (hTKV, hTLV, and hTKLV) and symptom burden (ADPKD-related pain and GI symptoms) were gender de- pendent, we tested the interaction between these characteristics. No significant interaction with gender was found, indicating that all associations could be tested across the complete study population and that stratification by gender was not necessary. hTKV was not associated with severity of ADPKD-related pain in the overall population $(\mathrm{R}=$ $0.05, p=0.44$; Fig. 1 ). In contrast, hTLV and hTKLV were both correlated with ADPKD-related pain $(\mathrm{R}=0.20, p<$ 0.001 and $\mathrm{R}=0.23, p<0.001$ ). After adjustment for disease severity, by correction for age, gender and eGFR, these associations remained significant $(\mathrm{R}=0.23, p<0.001$ and $\mathrm{R}=$ $0.20, p<0.001$, respectively). The regression analysis containing hTKLV was also more strongly associated with pain than the analysis containing hTKV $(p=0.04)$, whereas this was not the case for the analysis containing hTLV $(p=0.2)$. 
Table 2. Prevalence and severity of ADPKD-related pain and gastrointestinal symptoms overall and stratified for gender

\begin{tabular}{|c|c|c|c|c|}
\hline & $\begin{array}{l}\text { Overall } \\
(n=309)\end{array}$ & $\begin{array}{l}\text { Males } \\
(n=145)\end{array}$ & $\begin{array}{l}\text { Females } \\
(n=196)\end{array}$ & $p$ value \\
\hline \multicolumn{5}{|l|}{ History of pain } \\
\hline Renal-related pain & $147(47.6)$ & $63(43.2)$ & $84(51.5)$ & 0.14 \\
\hline Liver-related pain & $37(12.0)$ & $3(2.1)$ & $34(20.9)$ & $<0.001$ \\
\hline Renal- or liver-related pain & $157(50.8)$ & $66(45.2)$ & $91(55.8)$ & 0.06 \\
\hline \multicolumn{5}{|l|}{ Presence of pain } \\
\hline Renal-related pain & $77(24.9)$ & $28(19.2)$ & $49(30.1)$ & 0.04 \\
\hline Liver-related pain & $35(11.3)$ & $6(4.1)$ & $29(17.8)$ & $<0.001$ \\
\hline Renal- or liver-related pain & $85(27.5)$ & $29(19.9)$ & $56(34.4)$ & 0.006 \\
\hline \multicolumn{5}{|l|}{ Severity of pain in patients with pain } \\
\hline Renal-related pain & $4(3-6)$ & $3.5(3.0-6.0)$ & $5.0(3.0-6.0)$ & 0.3 \\
\hline Liver-related pain & $5(4-7)$ & $4(3.8-4.8)$ & $6(4-7)$ & 0.1 \\
\hline Renal or liver related pain & $4(3-7)$ & $4.0(3.0-6.0)$ & $5.0(3.0-7.0)$ & 0.2 \\
\hline \multicolumn{5}{|l|}{ Gastrointestinal symptoms } \\
\hline Lower abdominal pain & $46(14.9)$ & $14(9.6)$ & $32(19.6)$ & 0.02 \\
\hline Upper abdominal pain & $55(17.8)$ & $14(9.6)$ & $41(25.2)$ & $<0.001$ \\
\hline Heartburn & $70(22.7)$ & $33(22.6)$ & $37(22.7)$ & 0.9 \\
\hline Regurgitation & $57(18.4)$ & $26(17.8)$ & $31(19.0)$ & 0.9 \\
\hline Nausea & $42(13.6)$ & $10(6.8)$ & $32(19.6)$ & 0.001 \\
\hline Vomiting & $10(3.2)$ & $3(2.1)$ & $7(4.3)$ & 0.3 \\
\hline Loss of appetite & $50(16.2)$ & $15(10.3)$ & $35(21.5)$ & 0.01 \\
\hline Early satiety & $99(32.0)$ & $29(19.9)$ & $70(42.9)$ & $<0.001$ \\
\hline Dyspnea & $76(24.6)$ & $28(19.2)$ & $48(29.4)$ & 0.05 \\
\hline Increasing abdominal volume & $78(25.2)$ & $24(16.4)$ & $54(33.1)$ & 0.001 \\
\hline Involuntary weight loss & $9(2.9)$ & $2(1.4)$ & $7(4.3)$ & 0.1 \\
\hline \multicolumn{5}{|l|}{ Severity of present GI symptoms } \\
\hline GI-sum score & $12.0(8.0-21.0)$ & $9.0(4.5-16.7)$ & $17.6(15.2-23.1)$ & $<0.001$ \\
\hline
\end{tabular}

GI, gastrointestinal. Denominators depend on the number of patients who provided an answer for a specific question in the questionnaire.

Data is shown as $n(\%)$, median (interquartile range). Renal and liver pain measured on scale $1-10$ ( $1=$ no pain); GI-sum score ranging from $0-100(0=$ no symptoms $)$.

We then tested whether kidney and liver volume were associated with GI sum score. No association was found for hTKV $(\mathrm{R}=0.10, p=0.09)$, whereas hTLV and hTKLV were both associated with the GI sum score $(\mathrm{R}=0.23, p<$ 0.001 and $\mathrm{R}=0.23, p<0.001$, respectively; Fig. 2). Again, the association with GI symptoms was significantly stronger for the regression analysis containing hTKLV compared with the analysis containing hTKV $(p=$ 0.04 ) but not compared with the analysis with hTLV ( $p=$ $0.5)$.

Of note, we performed a sensitivity analysis to test whether these associations were different in patients with larger kidneys (hTKV $>1,000 \mathrm{~mL} / \mathrm{m}$ ). Essentially the same results were found as in the initial analysis; hTLV and hTKLV were, and hTKV was not, associated with ADPKD-related pain and GI symptoms.

Total Kidney Liver Volume in ADPKD

\section{Differences in Symptom Burden between Males and Females}

As a secondary goal, we assessed differences in symptom burden between men and women. Renal and liver pain were present in 30.1 and $17.8 \%$ of females, while this only accounted for 19.2 and $4.1 \%$ in males $(p=0.04$ and $p<0.001$, respectively). In case a patient experienced renal or liver pain, the severity of pain was similar among males and females. GI symptoms were more prevalent among females (Table 2). GI symptoms as expressed in the GI sum score were more severe in females than in males (17.6 vs. 9.0, $p<0.001)$.

Females had larger hTLV and smaller hTKV than males (hTLV: 1,249 [1,034-1,901] vs. 1,130 [967-1,336] $\mathrm{mL} / \mathrm{m}, p<0.001$ and hTKV: 923 [604-1,330] vs. 1,314 $[935-2,145] \mathrm{mL} / \mathrm{m}, p<0.001)$. hTKLV did not differ be- 


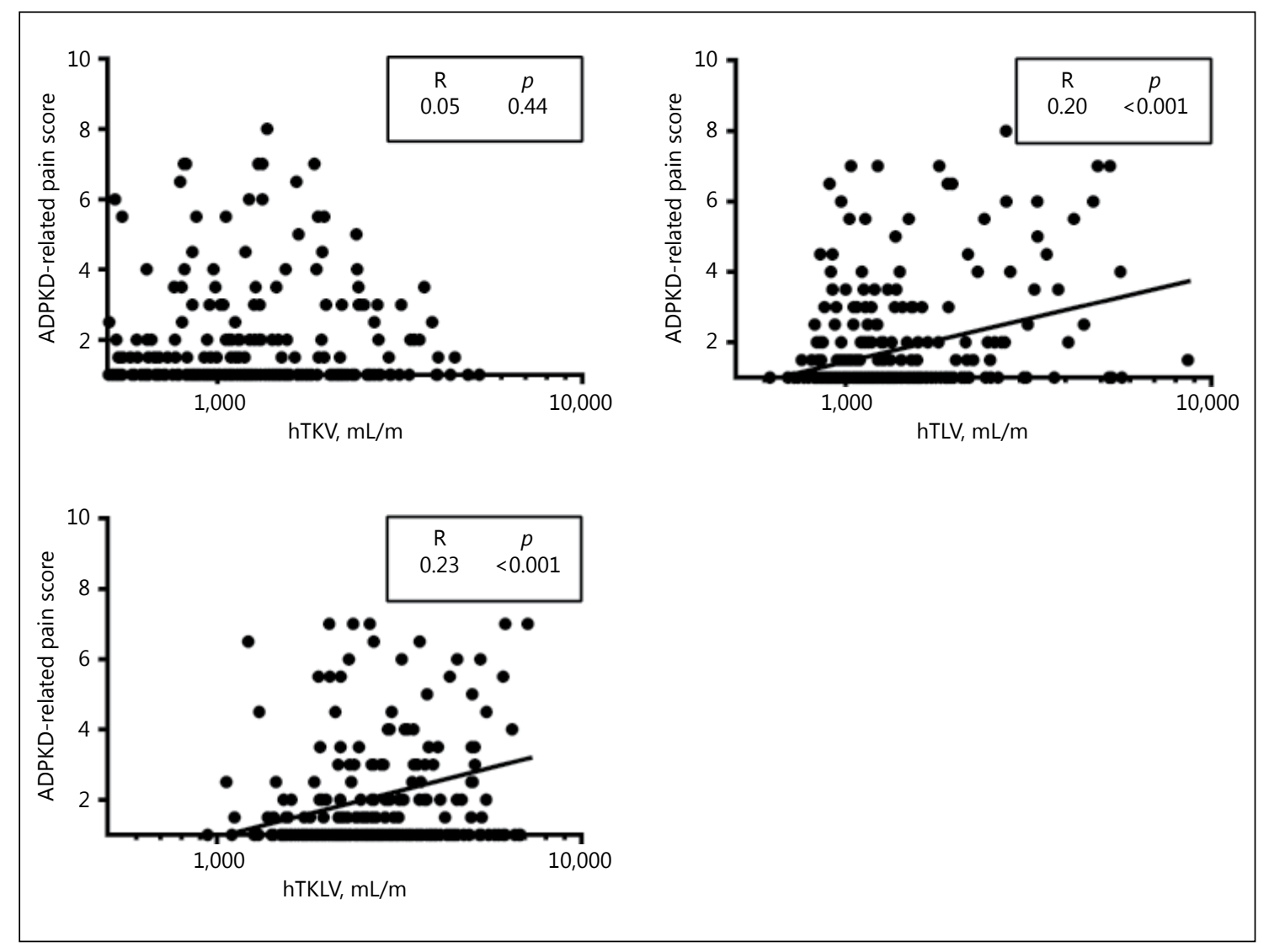

Fig. 1. Associations of height-adjusted TKV (hTKV), total liver volume (hTLV) and combined total kidney liver volume (hTKLV) with ADPKD-related pain score (1-10).

tween both genders (females: 2,424 [1,939-3,213] mL/m, males: $2,537[2,065-3,547] \mathrm{mL} / \mathrm{m}, p=0.2)$. Female gender was positively associated with symptom burden in ADPKD patients, but after adjustment for hTLV, this association lost significance.

\section{Height-Adjusted versus Non-Height-Adjusted Organ \\ Volumes}

No difference was observed in the association with symptoms between the regression analysis with either hTKV or TKV $(p=1.0)$, whereas the regression analysis with hTLV and hTKLV had stronger associations with pain than the regression analysis with TLV and TKLV $(p=$ 0.02 and $p=0.01$, respectively). For GI sum score, similar results were found. The analyses containing hTLV and hTKLV were more strongly associated with GI symptoms than non-height-adjusted regression analysis $(p=0.01$ and $p=0.01$, respectively), which did not account for the regression analysis containing hTKV $(p=1.0)$. Of note, the results of correlation analyses of ADPKD-related pain and
GI symptoms with non-height-adjusted TKV, TLV and TKLV were essentially similar to the results of the primary analyses with hTKV, hTLV and hTKLV (Table 3; online suppl. Table 2). The relations between organ volume and symptom burden still existed but were less strong compared to the height-adjusted regression analysis (Table 3).

\section{Discussion}

This study shows that both hTKLV and hTLV are moderately associated with pain and GI symptoms in patients with later stage ADPKD, while hTKV is not. Other patient-related characteristics, such as a history of urinary tract infection, renal cyst infection, liver cyst infection and macroscopic hematuria, were also associated with symptom burden. We found that females more frequently encountered symptoms than males. However, gender was not an effect modifier in the relation between organ volume and symptoms and the higher symptom burden in 


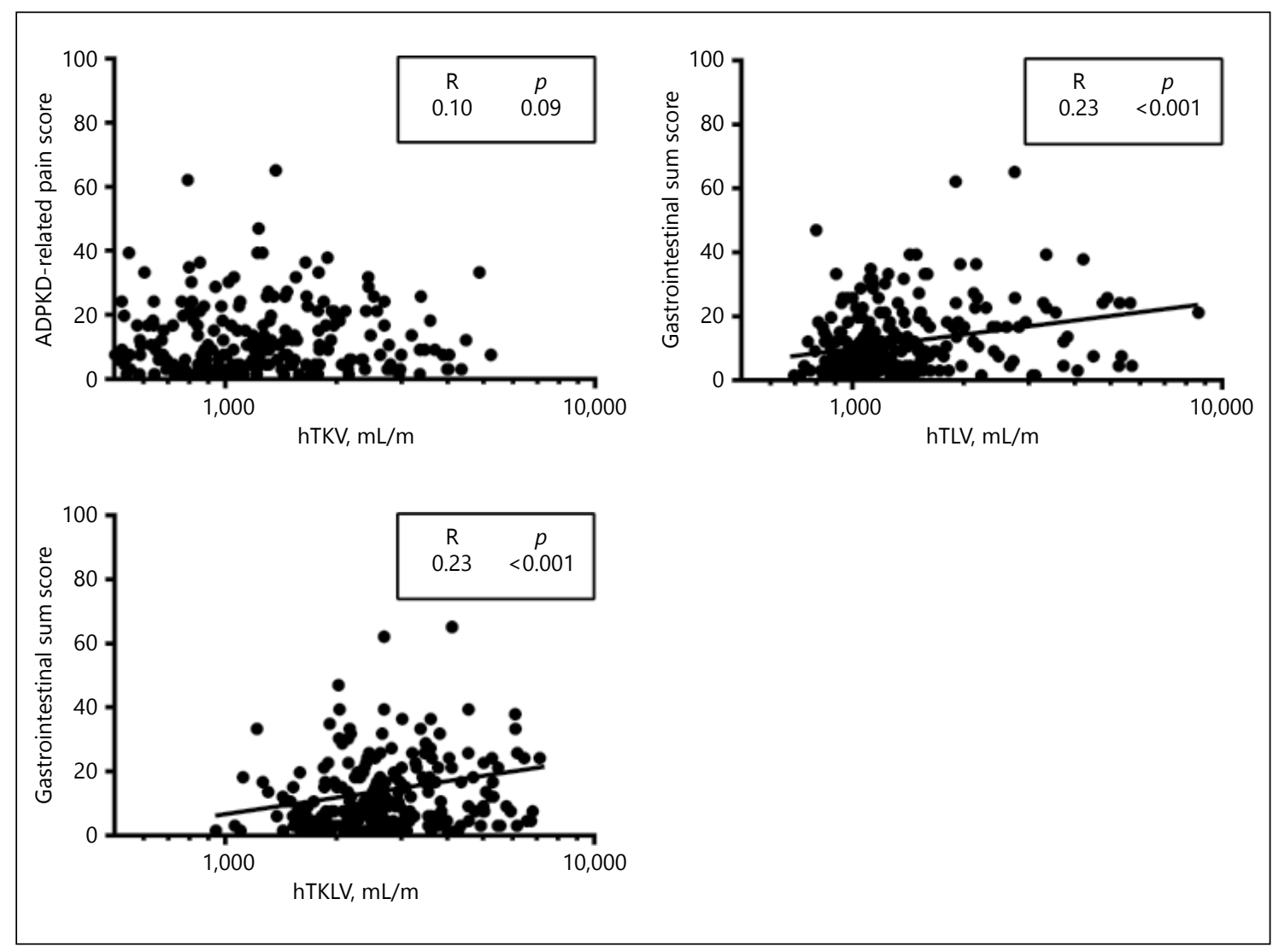

Fig. 2. Associations of height-adjusted TKV (hTKV), total liver volume (hTLV) and combined total kidney liver volume (hTKLV) with gastrointestinal sum score $(0-100)$.

women seems to be explained by their larger hTLV. In addition, analyses containing height-adjusted organ volumes were more strongly associated with pain and GI symptoms compared to non-height-adjusted analyses.

The general assumption is that a large kidney volume in ADPKD plays a role in causing pain [2]. Interestingly, 2 studies that investigated the association between kidney volume and pain did not confirm this assumption $[5,8]$. The authors found that TKV did not differ between those patients taking or not taking analgesics [8]. Only at extreme renal volumes in ADPKD ( $\mathrm{hTKV}>1,000 \mathrm{~mL} / \mathrm{m}$ ), an association between kidney volume and pain was found [5]. In our study, no association was found between $\mathrm{hTKV}$ and pain in the overall study population or in patients with very large kidneys. The present data adds to the evidence that the link between hTKV and pain is weak or even absent.

Previous studies found inconsistent results regarding the relation between liver volume and symptom burden. One study by Hogan et al. [10] that included patients with early stage ADPKD (eGFR $>60 \mathrm{~mL} / \mathrm{min} / 1.73 \mathrm{~m}^{2}$ ), found an association between liver volume and reduced quality of life. However, another study found no such relation in 92 patients with polycystic liver disease, of whom $67 \%$ had ADPKD [11]. Of note, this latter study included only patients with symptomatic polycystic liver disease. This study model is not suitable to assess possible associations between symptoms and liver volume. Our results suggest, in accordance with the results of Hogan et al. [10], that liver volume in ADPKD contributes significantly to symptom burden, as both hTLV and hTKLV were associated with pain and GI symptoms. The reason liver volume seems to play a more important role in causing symptoms than kidney volume cannot be concluded from the present data.

Only one previous study has investigated the role of combined total kidney liver volume on patient-reported outcome measures and found no association with healthrelated quality of life [7]. Of note, kidney and liver volumes were available in only 31 out of 219 included patients and the lack of significant associations may be due 
Table 3. Univariate associations of height-adjusted kidney and liver volumes with pain and gastrointestinal symptoms

\begin{tabular}{|c|c|c|c|c|c|c|}
\hline \multirow[t]{3}{*}{ Dependent variables } & \multicolumn{6}{|c|}{ Independent variables } \\
\hline & \multicolumn{2}{|l|}{ hTKV } & \multicolumn{2}{|c|}{ hTLV } & \multicolumn{2}{|c|}{ hTKLV } \\
\hline & $\mathrm{R}$ & $p$ value & $\mathrm{R}$ & $p$ value & $\mathrm{R}$ & $p$ value \\
\hline \multicolumn{7}{|l|}{ History of pain } \\
\hline Renal-related pain & 0.10 & 0.1 & 0.15 & 0.01 & 0.22 & $<0.001$ \\
\hline Liver-related pain & -0.06 & 0.3 & 0.30 & $<0.001$ & 0.20 & 0.001 \\
\hline Renal- or liver-related pain & 0.12 & 0.1 & 0.21 & $<0.001$ & 0.27 & $<0.001$ \\
\hline \multicolumn{7}{|l|}{ Presence of pain } \\
\hline Renal-related pain & 0.07 & 0.2 & 0.16 & 0.01 & 0.21 & $<0.001$ \\
\hline Liver-related pain & 0.01 & 0.8 & 0.25 & $<0.001$ & 0.21 & $<0.001$ \\
\hline Renal- or liver-related pain & 0.06 & 0.3 & 0.21 & $<0.001$ & 0.24 & $<0.001$ \\
\hline \multicolumn{7}{|l|}{ Severity of present pain } \\
\hline Renal-related pain & 0.04 & 0.5 & 0.14 & 0.02 & 0.17 & 0.003 \\
\hline Liver-related pain & 0.04 & 0.5 & 0.27 & $<0.001$ & 0.26 & $<0.001$ \\
\hline Renal- or liver-related pain & 0.05 & 0.4 & 0.20 & $<0.001$ & 0.23 & $<0.001$ \\
\hline \multicolumn{7}{|l|}{ Gastrointestinal symptoms } \\
\hline Lower abdominal pain & 0.06 & 0.3 & 0.10 & 0.1 & 0.09 & 0.1 \\
\hline Upper abdominal pain & 0.03 & 0.6 & 0.22 & $<0.001$ & 0.19 & 0.001 \\
\hline Heartburn & 0.15 & 0.01 & 0.05 & 0.4 & 0.13 & 0.03 \\
\hline Regurgitation & 0.12 & 0.03 & 0.14 & 0.02 & 0.15 & 0.01 \\
\hline Nausea & -0.04 & 0.5 & 0.22 & $<0.001$ & 0.11 & 0.05 \\
\hline Vomiting & -0.02 & 0.8 & 0.13 & 0.03 & 0.09 & 0.1 \\
\hline Loss of appetite & 0.01 & 0.8 & 0.18 & 0.002 & 0.16 & 0.01 \\
\hline Early satiety & 0.06 & 0.3 & 0.21 & $<0.001$ & 0.21 & $<0.001$ \\
\hline Dyspnea & 0.06 & 0.3 & 0.14 & 0.02 & 0.11 & 0.1 \\
\hline Increasing abdominal volume & 0.12 & 0.03 & 0.15 & 0.01 & 0.22 & $<0.001$ \\
\hline Involuntary weight loss & -0.02 & 0.7 & 0.08 & 0.2 & 0.00 & 1.0 \\
\hline \multicolumn{7}{|c|}{ Severity present gastrointestinal symptoms } \\
\hline GI-sum score & 0.10 & 0.1 & 0.23 & $<0.001$ & 0.23 & $<0.001$ \\
\hline
\end{tabular}

hTKV, height-adjusted total kidney volume; hTLV, height-adjusted total liver volume; hTKLV, height-adjusted total kidney liver volume; GI, gastrointestinal; R, correlation. hTKV, hTLV and hTKLV were log transformed. Denominators depend on the number of patients who provided an answer for a specific question in the questionnaire. Renal and liver pain measured on scale $1-10$ ( $1=$ no pain); GI-sum score ranging from $0-100(0=$ no symptoms). Significant $p$ values $(<0.05)$ are in bold.

to the small sample size. By contrast, we found significant association between hTKLV, hTLV and symptoms. It should be noted, however, that the strength of these associations was moderate. This suggests that symptom burden is multifactorial and that other factors may contribute [7]. Potential other determinants may include coping mechanisms and comorbidity, such as a history of urinary tract infection, renal cyst infection, liver cyst infection and macroscopic hematuria, which according to our results, were also related to current ADPKD-related symptom burden. Adequate management of these events may be indicated to reduce the presence of symptom burden in ADPKD.
In accordance with earlier studies, our data indicates a gender imbalance in prevalence and severity of ADPKDrelated pain and GI symptoms [5]. The same observation is true for the general population, where females report pain and GI symptoms more frequently [16-18]. Surprisingly, in our study, gender was no effect modifier in the relation between volumes and symptom burden. As expected, females had larger hTLV compared to males, and when adjusted for hTLV, variations in symptom burden between males and females disappeared. Based on these data we hypothesize that the higher symptom burden in women could be explained by their larger hTLV, though it might be that women experience more pain in general, compared to men. 
In symptomatic ADPKD patients, therapies are indicated that can slow cyst growth in both kidneys and liver. The TEMPO 3:4 trial demonstrated that tolvaptan, a vasopressin V2 receptor antagonist, decreased the rate of growth in TKV [26]. In contrast to the beneficial effect on renal cyst growth, tolvaptan presumably has no effect on liver cyst growth because the V2 receptor is not expressed in liver tissue. Our results suggest that in order to effectively reduce ADPKD-related symptom burden, therapy should also target liver cysts. Somatostatin analogues have been shown to reduce liver growth rate and symptoms in ADPKD patients with severe polycystic liver disease $[13,14,27]$. These agents also hold promise to reduce the rate of growth of TKV $[13,28]$ and the rate of renal function decline in ADPKD patients [29]. Somatostatin analogue therapy may therefore become a treatment option in ADPKD patients who undergo pain and experience GI symptoms, but this issue needs additional study before somatostatin analogues can be prescribed in clinical practice. The moderate association between kidneys and liver enlargement with symptoms suggests that symptom burden is multifactorial and that other factors may contribute to this condition. Two randomized controlled trials are ongoing to test the efficacy of somatostatin analogues to delay disease progression and reduce symptom burden in ADPKD [21,30].

A limitation of our study is that it we followed a crosssectional observational design. The effect of kidney and liver growth on symptoms could not be assessed. We performed a study in the setting of a randomized controlled trial with specific inclusion criteria for age (18-60 years) and renal function (eGFR $30-60 \mathrm{~mL} / \mathrm{min} / 1.73 \mathrm{~m}^{2}$ ). This may make extrapolation of our findings to the general ADPKD population difficult. It is clear that even ADPKD patients with eGFR CKD stages 1 or 2 can have ADPKDrelated pain and GI symptoms. However, we observed that neither ADPKD-related pain nor GI symptoms were associated with renal function, suggesting that our results may be valid for the ADPKD population as a whole. The main strength of our study is the systematic and prospective nature of data collection, which resulted in a wellphenotyped population.

In conclusion, we found that combined kidney and liver volume is associated with pain and GI symptoms in ADPKD, with a more prominent role for liver volume than for kidney volume. It should be noted, however, that other determinants, such as a history of urinary tract infection, renal cyst infection, liver cyst infection and macroscopic hematuria, also seem to be of importance in determining symptom burden in ADPKD. Height-adjusted organ volumes were more strongly associated with symptom burden compared to the non-height-adjusted organ volumes, emphasizing the relevance of height adjustment to assess associations with symptom burden. Female ADPKD patients more often experienced pain and GI symptoms than males. This gender difference could be explained by larger liver volumes in females compared to males. Lastly, our results implicate that efforts to reduce symptom burden should focus on liver volume.

\section{Acknowledgements}

\section{DIPAK Consortium}

The DIPAK Consortium is an inter-university collaboration in the Netherlands established to study ADPKD and to develop rational treatment strategies for this disease. The DIPAK Consortium is sponsored by the Dutch Kidney Foundation (grant CP10.12). Principal investigators are (in alphabetical order): J.P.H.D. (Department of Gastroenterology and Hepatology, Radboud University Medical Center Nijmegen), H.F. (Department Nephrology, Leiden University Medical Center), R.T.G. (Department of Nephrology, University Medical Center Groningen), D.J.M.P. (Department of Human Genetics, Leiden University Medical Center), J.F.M.W. (Department of Nephrology, Radboud University Medical Center Nijmegen), R.Z. (Department of Internal Medicine, Erasmus Medical Center Rotterdam).

\section{Financial Disclosure}

The authors have nothing to disclose.

\section{Disclosure Statement}

The authors have no conflicts of interest to declare.

\section{References}

1 Bae KT, Commean PK, Lee J: Volumetric measurement of renal cysts and parenchyma using MRI: phantoms and patients with polycystic kidney disease. J Comput Assist Tomogr 2000;24:614-619.

2 Grantham JJ, Mulamalla S, Swenson-Fields KI: Why kidneys fail in autosomal dominant polycystic kidney disease. Nat Rev Nephrol 2011;7:556-566.

3 Chapman AB, Devuyst O, Eckardt KU, Gansevoort RT, Harris T, Horie S, Kasiske BL, Odland D, Pei Y, Perrone RD, Pirson Y, Schrier RW, Torra R, Torres VE, Watnick T, Wheeler DC: Autosomal-dominant polycystic kidney disease (ADPKD): executive summary from a Kidney Disease: Improving Global Outcomes (KDIGO) controversies conference. Kidney Int 2015;88:17-27. 
4 Gevers TJ, Drenth JP: Diagnosis and management of polycystic liver disease. Nat Rev Gastroenterol Hepatol 2013;10:101-108.

5 Miskulin DC, Abebe KZ, Chapman AB, Perrone RD, Steinman TI, Torres VE, Bae KT, Braun W, Winklhofer FT, Hogan MC, Rahbari-Oskoui F, Moore CG, Flessner MF, Schrier RW; HALT-PKD Study: Health-related quality of life in patients with autosomal dominant polycystic kidney disease and CKD stages 1-4: a cross-sectional study. Am J Kidney Dis 2014;63:214-226.

6 Casteleijn NF, Visser FW, Drenth JP, Gevers TJ, Groen GJ, Hogan MC, Gansevoort RT; DIPAK Consortium: A stepwise approach for effective management of chronic pain in autosomal-dominant polycystic kidney disease. Nephrol Dial Transplant 2014;29(suppl 4):iv142-iv153.

7 Suwabe T, Ubara Y, Mise K, Kawada M, Hamanoue S, Sumida K, Hayami N, Hoshino J, Hiramatsu R, Yamanouchi M, Hasegawa E, Sawa N, Takaichi K: Quality of life of patients with ADPKD-Toranomon PKD QOL study: cross-sectional study. BMC Nephrol 2013;14 179 .

8 Rizk D, Jurkovitz C, Veledar E, Bagby S, Baumgarten DA, Rahbari-Oskoui F, Steinman T, Chapman AB: Quality of life in autosomal dominant polycystic kidney disease patients not yet on dialysis. Clin J Am Soc Nephrol 2009;4:560-566.

9 Kim H, Park HC, Ryu H, Kim K, Kim HS, Oh $\mathrm{KH}$, Yu SJ, Chung JW, Cho JY, Kim SH, Cheong HI, Lee K, Park JH, Pei Y, Hwang YH, Ahn C: Clinical correlates of mass effect in autosomal dominant polycystic kidney disease. PLoS One 2015;10:e0144526.

10 Hogan MC, Abebe K, Torres VE, Chapman $A B$, Bae KT, Tao C, Sun H, Perrone RD, Steinman TI, Braun W, Winklhofer FT, Miskulin DC, Rahbari-Oskoui F, Brosnahan G, Masoumi A, Karpov IO, Spillane S, Flessner M, Moore CG, Schrier RW: Liver involvement in early autosomal-dominant polycystic kidney disease. Clin Gastroenterol Hepatol 2015;13: 155-164.e6.

11 Wijnands TF, Neijenhuis MK, Kievit W, Nevens F, Hogan MC, Torres VE, Gevers TJ, Drenth JP: Evaluating health-related quality of life in patients with polycystic liver disease and determining the impact of symptoms and liver volume. Liver Int 2014;34:1578-1583.

12 Irazabal MV, Rangel LJ, Bergstralh EJ, Osborn SL, Harmon AJ, Sundsbak JL, Bae KT, Chapman AB, Grantham JJ, Mrug M, Hogan MC, El-Zoghby ZM, Harris PC, Erickson BJ, King BF, Torres VE; CRISP Investigators: Im- aging classification of autosomal dominant polycystic kidney disease: a simple model for selecting patients for clinical trials. J Am Soc Nephrol 2015;26:160-172.

13 van Keimpema L, Nevens F, Vanslembrouck R, van Oijen MG, Hoffmann AL, Dekker HM de Man RA, Drenth JP: Lanreotide reduces the volume of polycystic liver: a randomized, double-blind, placebo-controlled trial. Gastroenterology 2009;137:1661-1668.e1-e2.

14 Gevers TJ, Hol JC, Monshouwer R, Dekker HM, Wetzels JF, Drenth JP: Effect of lanreotide on polycystic liver and kidneys in autosomal dominant polycystic kidney disease: an observational trial. Liver Int 2015;35:16071614.

15 Van Keimpema L, De Koning DB, Van Hoek B, Van Den Berg AP, Van Oijen MG, De Man RA, Nevens F, Drenth JP: Patients with isolated polycystic liver disease referred to liver centres: clinical characterization of 137 cases. Liver Int 2011;31:92-98.

16 Bartley EJ, Fillingim RB: Sex differences in pain: a brief review of clinical and experimental findings. Br J Anaesth 2013;111:52-58.

17 Mifflin KA, Kerr BJ: The transition from acute to chronic pain: understanding how different biological systems interact. Can J Anaesth 2014;61:112-122.

18 Tielemans MM, Jaspers Focks J, van Rossum LG, Eikendal T, Jansen JB, Laheij RJ, van Oijen MG: Gastrointestinal symptoms are still prevalent and negatively impact healthrelated quality of life: a large cross-sectional population based study in The Netherlands. PLoS One 2013;8:e69876.

19 Drenth JP, Chrispijn M, Nagorney DM, Kamath PS, Torres VE: Medical and surgical treatment options for polycystic liver disease. Hepatology 2010;52:2223-2230.

20 Pei Y, Obaji J, Dupuis A, Paterson AD, Magistroni R, Dicks E, Parfrey P, Cramer B, Coto E, Torra R, San Millan JL, Gibson R, Breuning M, Peters D, Ravine D: Unified criteria for ultrasonographic diagnosis of ADPKD. J Am Soc Nephrol 2009;20:205-212.

21 Meijer E, Drenth JP, d'Agnolo H, Casteleijn NF, de Fijter JW, Gevers TJ, Kappert P, Peters DJ, Salih M, Soonawala D, Spithoven EM, Torres VE, Visser FW, Wetzels JF, Zietse R, Gansevoort RT; DIPAK Consortium: Rationale and design of the DIPAK 1 study: a randomized controlled clinical trial assessing the efficacy of Lanreotide to halt disease progression in autosomal dominant polycystic kidney disease. Am J Kidney Dis 2014;63:446-455.

22 Gerbershagen HJ, Rothaug J, Kalkman CJ, Meissner W: Determination of moderate-to- severe postoperative pain on the numeric rating scale: a cut-off point analysis applying four different methods. Br J Anaesth 2011; 107:619-626.

23 van Marrewijk CJ, Mujakovic S, Fransen GA, Numans ME, de Wit NJ, Muris JW, van Oijen MG, Jansen JB, Grobbee DE, Knottnerus JA, Laheij RJ: Effect and cost-effectiveness of step-up versus step-down treatment with antacids, H2-receptor antagonists, and proton pump inhibitors in patients with new onset dyspepsia (DIAMOND study): a primarycare-based randomised controlled trial. Lancet 2009;373:215-225.

24 Levey AS, Stevens LA, Schmid CH, Zhang YL, Castro AF 3rd, Feldman HI, Kusek JW, Eggers P, Van Lente F, Greene T, Coresh J; CKDEPI (Chronic Kidney Disease Epidemiology Collaboration): A new equation to estimate glomerular filtration rate. Ann Intern Med 2009;150:604-612.

25 Cameron AC, Windmeijer FAG: R-squared measures for count data regression models with applications to health-care utilization. J Bus Econ Stat 1996;14:209-220.

26 Torres VE, Chapman AB, Devuyst O, Gansevoort RT, Grantham JJ, Higashihara E, Perrone RD, Krasa HB, Ouyang J, Czerwiec FS; TEMPO 3:4 Trial Investigators: Tolvaptan in patients with autosomal dominant polycystic kidney disease. N Engl J Med 2012;367:24072418.

27 Caroli A, Antiga L, Cafaro M, Fasolini G, Remuzzi A, Remuzzi G, Ruggenenti P: Reducing polycystic liver volume in ADPKD: effects of somatostatin analogue octreotide. Clin J Am Soc Nephrol 2010;5:783-789.

28 Hogan MC, Masyuk TV, Page LJ, Kubly VJ, Bergstralh EJ, Li X, Kim B, King BF, Glockner J, Holmes DR 3rd, Rossetti S, Harris PC, LaRusso NF, Torres VE: Randomized clinical trial of long-acting somatostatin for autosomal dominant polycystic kidney and liver disease. J Am Soc Nephrol 2010;21:1052-1061.

29 Caroli A, Perico N, Perna A, Antiga L, Brambilla P, Pisani A, Visciano B, Imbriaco $M$, Messa P, Cerutti R, Dugo M, Cancian L, Buongiorno E, De Pascalis A, Gaspari F, Carrara F, Rubis N, Prandini S, Remuzzi A, Remuzzi G, Ruggenenti P; ALADIN Study Group: Effect of longacting somatostatin analogue on kidney and cyst growth in autosomal dominant polycystic kidney disease (ALADIN): a randomised, placebo-controlled, multicentre trial. Lancet 2013;382:1485-1495.

30 Lanreotide in Polycystic Kidney Disease Study (LIPS). https://clinicaltrials.gov/ct2/ show/NCT02127437. 